\title{
DEVELOPMENT OF CIRCUITS AND SYSTEM MODELS FOR THE SYNCHRONIZATION OF THE ILC CRAB CAVITIES
}

\author{
G. Burt, R. Carter, A. Dexter, R. Jenkins, I. Tahir, Cockcroft Institute (CI), Lancaster University, \\ Lancaster, LA1 4YR, UK \\ C. Beard, P. Goudket, A. Kalinin, L. Ma, P. McIntosh, ASTeC/CI, STFC, Daresbury WA4 4AD, UK
}

\section{Abstract}

The ILC reference design report (RDR) recommends a $14 \mathrm{mrad}$ crossing angle for the positron and electron beams at the IP. A matched pair of crab cavity systems are required in the beam delivery system to align both bunches at the IP. The use of a multi-cell, 3.9 GHz dipole mode superconducting cavity is proposed, derived from the Fermilab CKM cavity being developed as a beam slice diagnostic [1]. Dipole-mode cavities phased for crab rotation are shifted by $90^{\circ}$ with respect to similar cavities phased for deflection. Uncorrelated phase errors of $0.086^{\circ}$ (equivalent to $61 \mathrm{fs}$ ) for the two cavity systems, gives an average of $180 \mathrm{~nm}$ for the relative deflection of the bunch centers. For a horizontal bunch size $\sigma_{\mathrm{x}}=655 \mathrm{~nm}$, a deflection of $180 \mathrm{~nm}$ reduces the ILC luminosity by $2 \%$. The crab cavity systems are to be placed $\sim 30 \mathrm{~m}$ apart and synchronization to within $61 \mathrm{fs}$ is required; this is on the limit of what is presently achievable. This paper describes LLRF circuits under development at the Cockcroft Institute for proof of principle experiments planned on the ERLP at Daresbury and on the ILCTA test beamline at FNAL. Simulation results for stabilisation performance are also given.

\section{PRELIMINARY LLRF LAYOUT}

There are a wide range of possible technologies and configurations that might be used for the crab cavity LLRF system. The circuits being developed are for proof of principle. Important criteria for the current system are flexibility and accurate synchronisation in the absence of beam based calibration. For superconducting cavities, the microphonics which act to spoil phase synchronism are independent for each cavity. Accordingly we chose separate amplifiers and controllers for each cavity. In order to synchronize the crab cavities we must therefore provide accurately synchronized timing signals very close to the output couplers of each cavity. The cavities then need to be stabilized with respect to the local timing signal. The timing error between cavity phases has three components, one from the synchronization of the timing signals and two from the cavity to timing signal synchronization. Our initial target is a cavity to a timing signal synchronization of $20 \mathrm{fs}$ and a timing signal to timing signal synchronization also of $20 \mathrm{fs}$. Timing synchronization of $10 \mathrm{fs}$ has recently been claimed at LBLN using mode locked lasers [2]. Our current system uses an RF interferometer which is not expected to achieve this performance but which could be easily replaced with such a system. The development system sketched in figure 1 employs digital phase detection permitting absolute measurement with minimal calibration issues, 16 bit A to D conversion at 100 MSPS, DSP control algorithm implementation giving maximum flexibility and IQ modulation of the RF drive.

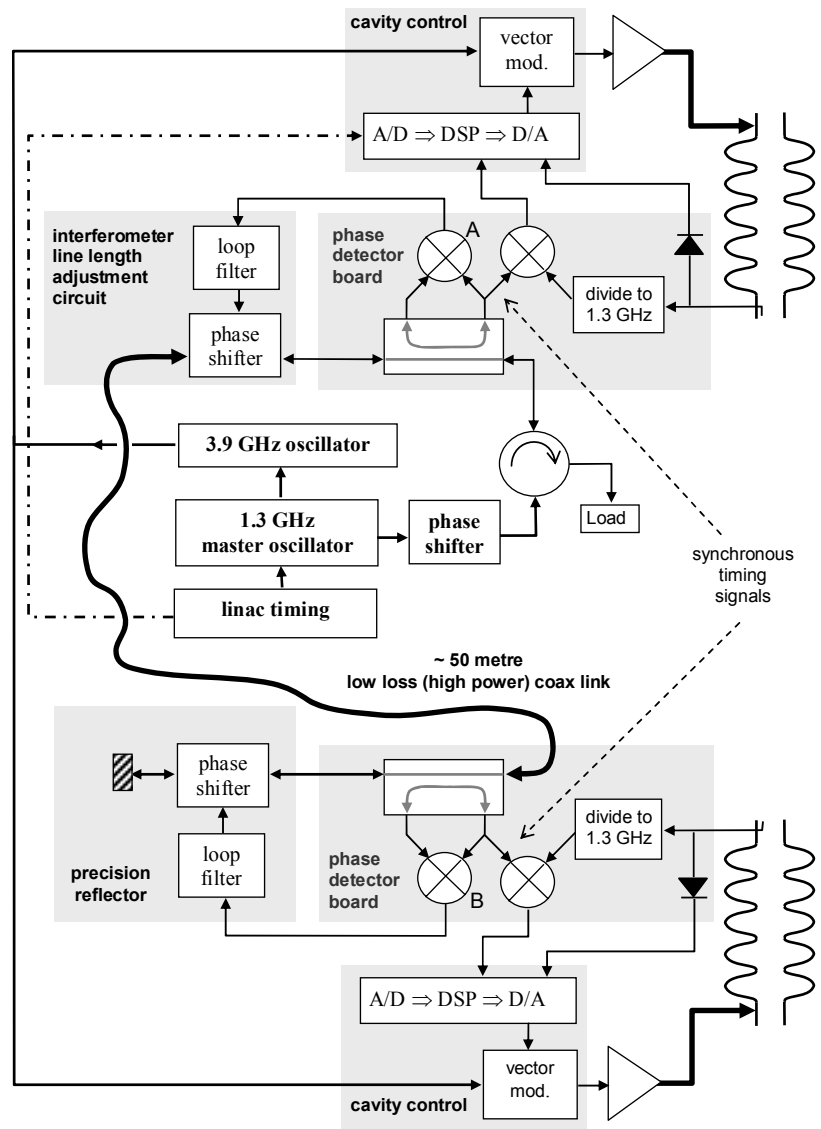

Figure 1: Synchronization layout.

\section{CAVITY MODEL}

The accuracy to which the phase of each cavity can be stabilized against unpredictable components of the disturbing influences of microphonics and beam-loading, will depend on how accurately the phase can be measured and the maximum control system gain consistent with stability. High gain RF control stability of the TESLA cavities has recently been discussed by Vogel [3]. The gain stability limit for the control system will depend on loop delay. For an analogue control loop, delay will arise from input filtering and amplifier bandwidth. For the digital control system being proposed here, control loop delay depends additionally on ADC, DAC and DSP processing time. Compensation for the additional loop delay of a digital controller is made by opportunities for sophisticated input filtering, real time variation of control parameters and anticipation of repetitive disturbances. 
Calculation indicates that to get the required performance, digital processing delay needs to be less than about $1 \mu \mathrm{s}$.

The anticipated performance of the control system can be modelled numerically. We apply the standard equations for cavity filling and numerically integrate the envelope equations [4] for anticipated microphonics and worst case beam-loading. Figure 2 shows the effective (diagonalized) equivalent circuit for a multi-cell cavity driven via a coupler. Values $\mathrm{L}_{\mathrm{i}}, \mathrm{R}_{\mathrm{i}}, \mathrm{C}_{\mathrm{i}}$ are chosen so that each parallel resonator represents a cavity mode and are determined from modal frequencies, $\mathrm{Q}$ and $\mathrm{R} / \mathrm{Q}$ values.

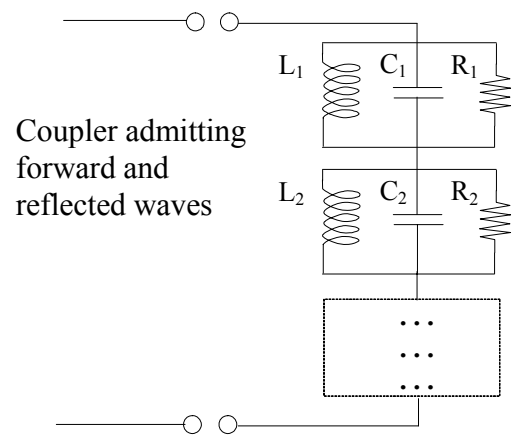

Figure 2: Cavity model.

One therefore solves

$\frac{\mathrm{d}^{2} \mathrm{~V}_{\mathrm{m}}}{\mathrm{dt}^{2}}+\frac{\omega_{\mathrm{m}}}{\mathrm{Q}_{\mathrm{om}}} \frac{\mathrm{dV}_{\mathrm{m}}}{\mathrm{dt}}+\frac{1}{\mathrm{Q}_{\mathrm{em}}} \omega_{\mathrm{m}} \sum_{\mathrm{j}=1}^{\mathrm{N}} \frac{\mathrm{dV}_{\mathrm{j}}}{\mathrm{dt}}+\omega_{\mathrm{m}}^{2} \mathrm{~V}_{\mathrm{m}}=\frac{2 \omega_{\mathrm{m}}}{\mathrm{Q}_{\mathrm{em}}} \frac{\mathrm{d}}{\mathrm{dt}}\{F \exp (-\mathrm{j} \omega \mathrm{t})\}$ where $\mathrm{V}_{\mathrm{m}}, \omega_{\mathrm{m}}, \mathrm{Q}_{\mathrm{om}}$ and $\mathrm{Q}_{\mathrm{em}}$ are the voltage, frequency, unloaded $\mathrm{Q}$ and $\mathrm{Q}$ external for the $\mathrm{m}^{\text {th }}$ mode respectively and $F$ is the amplitude of the forward wave in the coupler, $\omega$ is the drive frequency. This set of equations must be solved numerically as the modal frequencies are functions of time as determined by microphonics and the modal voltages take a step change each time a bunch passes through the cavity. These equations cannot be accurately integrated over the fill time and bunch train time which amounts to at least $10^{7} \mathrm{RF}$ cycles. Instead one solves for the real and imaginary parts of an amplitude function [4] determined by the equation

$$
\mathrm{V}_{\mathrm{m}}(\mathrm{t})=\left\{\mathrm{A}_{\mathrm{mr}}(\mathrm{t})+\mathrm{j} \mathrm{A}_{\mathrm{mi}}(\mathrm{t})\right\} \exp \{-\mathrm{j} \omega \mathrm{t}\}
$$

After neglecting second time derivatives of the slowly varying amplitude functions $A_{m r}$ and $A_{r i}$ and also terms of $\operatorname{order}\left(1 / \mathrm{Q}_{\mathrm{e}}\right)^{2}$ one obtains the envelope equations

$$
\begin{aligned}
\frac{\dot{\mathrm{A}}_{\mathrm{mr}}}{\omega_{\mathrm{m}}}+\frac{1}{4 \mathrm{Q}_{\mathrm{om}}}\left(\frac{\omega_{\mathrm{m}}^{2}}{\omega^{2}}+1\right) \mathrm{A}_{\mathrm{mr}}+\frac{1}{4 \mathrm{Q}_{\mathrm{em}}} \sum_{\mathrm{j}=1}^{\mathrm{N}}\left(\frac{\omega_{\mathrm{j}}^{2}}{\omega^{2}}+1\right) \mathrm{A}_{\mathrm{jr}} \\
-\left(\frac{\omega_{\mathrm{m}}}{\omega}-\frac{\omega}{\omega_{\mathrm{m}}}\right) \frac{\mathrm{A}_{\mathrm{mi}}}{2}=-\frac{1}{\mathrm{Q}_{\mathrm{em}}}\left(\frac{\dot{F}_{\mathrm{i}}}{\omega}-F_{\mathrm{r}}\right) \\
\frac{\dot{\mathrm{A}}_{\mathrm{mi}}}{\omega_{\mathrm{m}}}+\frac{1}{4 \mathrm{Q}_{\mathrm{om}}}\left(\frac{\omega_{\mathrm{m}}^{2}}{\omega^{2}}+1\right) \mathrm{A}_{\mathrm{mi}}+\frac{1}{4 \mathrm{Q}_{\mathrm{em}}} \sum_{\mathrm{j}=1}^{\mathrm{N}}\left(\frac{\omega_{\mathrm{j}}^{2}}{\omega^{2}}+1\right) \mathrm{A}_{\mathrm{ji}} \\
+\left(\frac{\omega_{\mathrm{m}}}{\omega}-\frac{\omega}{\omega_{\mathrm{m}}}\right) \frac{\mathrm{A}_{\mathrm{mr}}}{2}=\frac{1}{\mathrm{Q}_{\mathrm{em}}}\left(\frac{\dot{F}_{\mathrm{r}}}{\omega}+F_{\mathrm{i}}\right)
\end{aligned}
$$

For the planned 9 cell cavity, modes will be designated as mode $=\pi(1-\mathrm{m} / 9)$ so that $\mathrm{m}=0$ gives the $\pi$ mode.

The real and imaginary parts of the forward wave are determined by the controller which must reduce $\mathrm{A}_{0 \mathrm{i}}$ to zero and hold $\mathrm{A}_{0 \mathrm{r}}$ at a steady level $\mathrm{V}_{\mathrm{sp}}$ as appropriate for required kick. At this stage we have no detailed knowledge of system disturbances hence use of a proportional integral controller is appropriate for the model. Explicitly we take

$$
\begin{aligned}
& F_{\mathrm{r}}\left(\mathrm{t}+\mathrm{t}_{\text {delay }}\right)=\mathrm{c}_{\mathrm{pr}}\left(\mathrm{V}_{\mathrm{sp}}-\overline{\sum_{\mathrm{m}} \mathrm{A}_{\mathrm{mr}}}\right)+\mathrm{c}_{\mathrm{ir}} \int_{-\infty}^{\mathrm{t}} \mathrm{dt}\left(\mathrm{V}_{\mathrm{sp}}-\overline{\sum_{\mathrm{m}} \mathrm{A}_{\mathrm{mr}}}\right) \\
& F_{\mathrm{i}}\left(\mathrm{t}+\mathrm{t}_{\text {delay }}\right)=-\mathrm{c}_{\mathrm{pi}} \overline{\sum_{\mathrm{m}} \mathrm{A}_{\mathrm{mi}}}-\mathrm{c}_{\mathrm{ii}} \int_{-\infty}^{\mathrm{t}} \mathrm{dt} \overline{\sum_{\mathrm{m}} \mathrm{A}_{\mathrm{mi}}}
\end{aligned}
$$

where $c_{\mathrm{pr}}, \mathrm{c}_{\mathrm{pi}}, \mathrm{c}_{\mathrm{ir}}$ and $\mathrm{c}_{\mathrm{ii}}$ are the controller coefficients and $t_{\text {delay }}$ represents time delays in the digital processor. One wishes to control the $\pi$ mode, however the measured cavity voltage will be some weighted time average of the summed excitation of the modes. As the weightings for this average have yet to be determined, the modes are sampled with equal weight through a low pass filter having a time constant equal to the sampling rate. The amplifier is modelled as a second order filter with a time constant determined from its bandwidth. Beamloading of the $\pi$ mode with the ILC time structure is included. Wakefield calculations have shown that excitation of other modes by the beam is small. Monochromatic cavity vibration appropriate to the most prominent frequency measure on prototype CKM cavities is included.

\section{RESULTS}

Figures 3-7 show cavity voltage, drive amplitude and cavity phase during cavity filling and the passage of an ILC bunch train with an offset of $0.6 \mathrm{~mm}$ after 4.5 million $3.9 \mathrm{GHz}, \mathrm{RF}$ cycles. In this calculation only the cavity $\pi$ mode is modelled. Phase measurement errors are not included. The cavity has a microphonic frequency of $230 \mathrm{~Hz}$ which shifts the RF frequency by $600 \mathrm{~Hz}$. The "in phase" drive amplitude follows the beam loading whilst the "phase quadrature" drive amplitude compensates for microphonics as expected. If the beam offset is permitted to oscillate, then for perfect bunch timing the phase response remains as in figure 7 . Figure 8 shows the influence of bunch timing errors when the bunch offset oscillates at $2 \mathrm{kHz}$. Figures 9 and 10 show the effect of including the $8 \pi / 9$ mode at $+2.2 \mathrm{MHz}$ and the $7 \pi / 9$ mode at $+8.9 \mathrm{MHz}$ in addition to the $\pi$ mode at $3.9 \mathrm{GHz}$. Interestingly, beam-loading is seen to couple to the phase error when additional modes are included in the model. Note that the amplifier bandwidth was taken as $10 \mathrm{MHz}$. The gain was $36 \%$ below the point of instability [5]. Figure 10 represents a worst case control scenario in the absence of phase measurement errors. Using digital phase detectors and a loop bandwidth appropriate to the model, the measurement jitter after division to $1.3 \mathrm{GHz}$ will be of the order of 10 milli-degrees. 


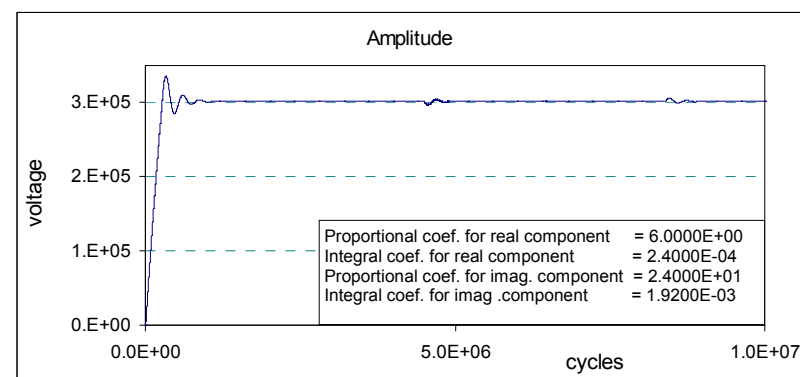

Figure 3 One mode and steady $0.6 \mathrm{~mm}$ beam offset

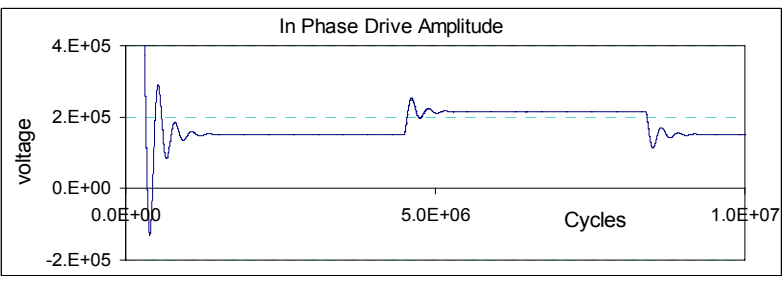

Figure 4 One mode and steady $0.6 \mathrm{~mm}$ beam offset

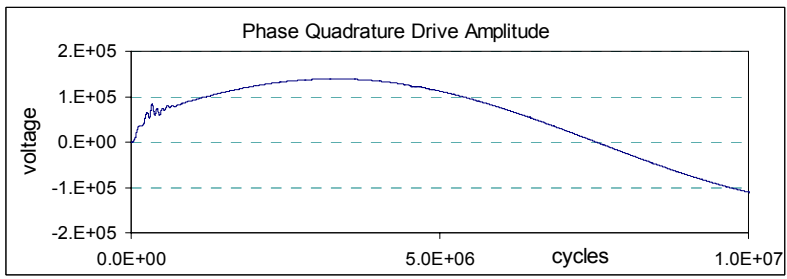

Figure 5 One mode and steady $0.6 \mathrm{~mm}$ beam offset

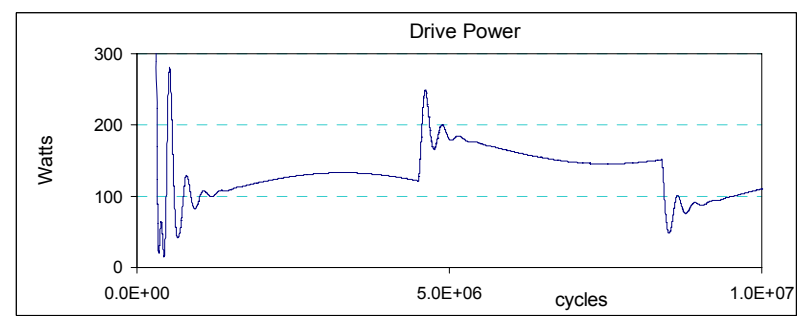

Figure 6 One mode and steady $0.6 \mathrm{~mm}$ beam offset

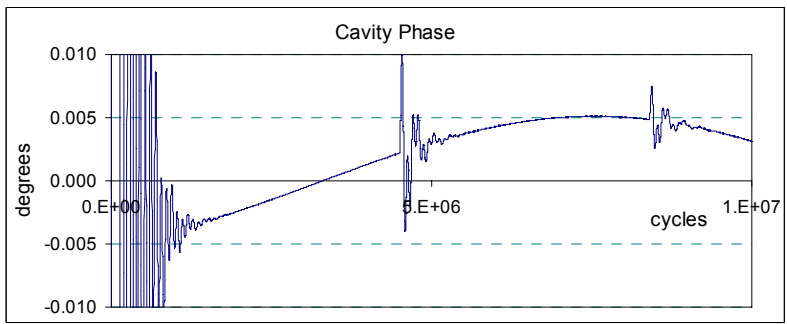

Figure 7 One mode and steady $0.6 \mathrm{~mm}$ beam offset

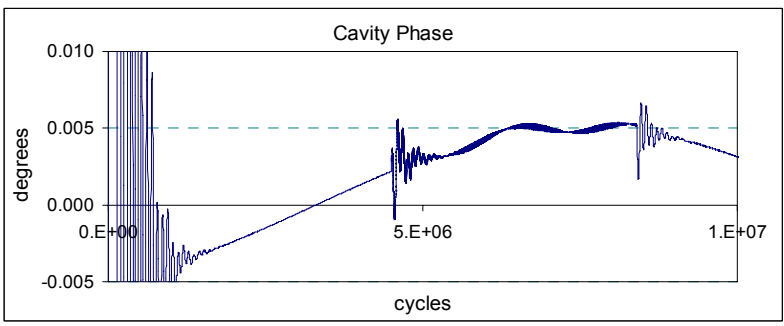

Figure 8 One mode and $0.6 \mathrm{~mm}$ oscillating beam offset and with 712 fs random, bunch timing errors

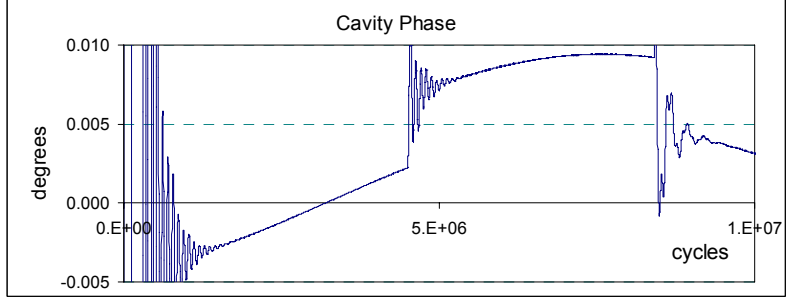

Figure 9 Three modes and $0.6 \mathrm{~mm}$ beam offset

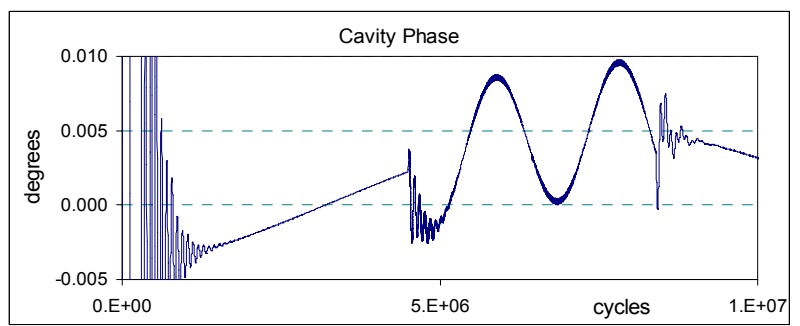

Figure 10 Three modes and $0.6 \mathrm{~mm}$ oscillating beam offset with 712 fs random, bunch timing errors

Modelling has also been undertaken with measurements errors included and to a rough approximation they can be simply added to the results displayed. Taking contributions together, stabilization to $20 \mathrm{fs} \sim 30$ millidegrees looks feasible.

\section{FUTURE WORK}

The LLRF circuits are soon to be tested by driving two adjacent cavities, independently in a vertical cryostat. As the cavities are adjacent it will be possible to make an independent measurement of relative cavity phase jitter.

\section{ACKNOWLEDGEMENTS}

The authors would like to thank L. Bellantoni for his helpful comments and provision of data for this study which is taking place under the envelope of the ILC crab cavity collaboration that includes FNAL as a major contributor.

The work here has been supported in part by the EC under Contract no.011899 RIDS and in part by UK Science and Technology Facilities Council (STFC).

\section{REFERENCES}

[1] M. McAshan, R. Wanzenberg, RF Design of a Transverse Mode Cavity for Kaon Separation, FERMILAB-TM-2144, May 2001.

[2] S. Lidia, "Progress in high precision timing and synchronization at LBNL", ERL07, Daresbury 2007

[3] E. Vogel, "High Gain proportional rf control stability at TESLA cavities", Phys Rev. Special Topics Accelerators and Beams 10, 052001 (2007)

[4] H. Henke, M. Filtz, "Envelope equations for transients in linear chains of resonators", PAC 1993

[5] H. Imsieke, A. Kholdyni, S.N. Simrock, "Time delay Compensation for the digital RF control at the TESLA test facility" LINAC 98 